\title{
Poorly differentiated clusters (PDC) in colorectal cancer: what is and ought to be known
}

\author{
Luca Reggiani Bonetti ${ }^{1}$, Valeria Barresi ${ }^{2}$, Stefania Bettelli ${ }^{1}$, Federica Domati ${ }^{3}$ and Cristian Palmiere ${ }^{4^{*}}$
}

\begin{abstract}
Background: The counting of poorly differentiated clusters of 5 or more cancer cells lacking a gland-like structure in a tumor mass has recently been identified among the histological features predictive of poor prognosis in colorectal cancer.

Main body: Poorly differentiated clusters can easily be recognized in the histological sections of colorectal cancer routinely stained with haematoxylin and eosin. Despite some limitations related to specimen fragmentation, counting can also be assessed in endoscopic biopsies. Based on the number of poorly differentiated clusters that appear under a microscopic field of a $\times 20$ objective lens (i.e., a microscopic field with a major axis of $1 \mathrm{~mm}$ ), colorectal cancer can be graded into malignancies as follows: tumors with $<5$ clusters as grade 1 , tumors with 5 to 9 clusters as grade 2 , and tumors with $\geq 10$ clusters as grade 3 . High poorly differentiated cluster counts are significantly associated with peri-neural and lympho-vascular invasion, the presence of nodal metastases or micrometastases, as well as shorter overall and progression free survival to colorectal cancer.
\end{abstract}

Conclusion: The morphological aspects and clinical relevance of poorly differentiated clusters counting in colorectal cancer are discussed in this review.

Keywords: Poorly differentiated clusters, Colorectal cancer, Tumor grading

\section{Background}

The incidence of colorectal cancer (CRC) has gradually increased over the decades in developed countries, and is now the third most-commonly diagnosed cancer [1-4].

In histopathological routine practice, tumor grade represents one of the most important predictive factors of colorectal cancer (CRC) aggressiveness [5]. To date, the most widely used system of defining CRC's histological grade is based on the percentage of tumor glands forming the mass. However, this system suffers from significant inter-observer variability due to the lack of objective methods with which to assess the amount of glandular components [6].

More recently, a novel histological grading system has been highlighted as a histopathological prognosticator of CRC. This system considers poorly differentiated

\footnotetext{
* Correspondence: cristian.palmiere@chuv.ch

${ }^{4}$ CURML, chemin de la Vulliette 4, 1000, Lausanne 25, Switzerland

Full list of author information is available at the end of the article
}

clusters (PDCs) composed of $\geq 5$ cancer cells present at invasive front of the tumor that lack full glandular formation, $[1,4,7-9]$.

\section{Main text}

PDC can be evaluated in representative haematoxylin and eosin (H\&E) stained slides that include the advancing edge of the tumor counted in the microscopic field under a $\times 20$ objective lens (i.e. a $1 \mathrm{~mm}$ microscopic field). CRC can then be categorized into three grades of malignancy based on the highest PDC count: grade 1 (G1) count less than 5 (Fig. 1a), grade 2 (G2) range between 5 and 9 (Fig. 1b) and grade 3 (G3) 10 or more PDC clusters found (Fig. 1c).

Although PDC and tumor budding are similar in morphology given that neither have gland silhouettes, they represent two different entities. Indeed, by definition, tumor budding foci are smaller than PDC and made up of isolated cancer cells or small clusters of fewer than 5 elements $[7,10]$. Thus, PDC are more easily recognizable 

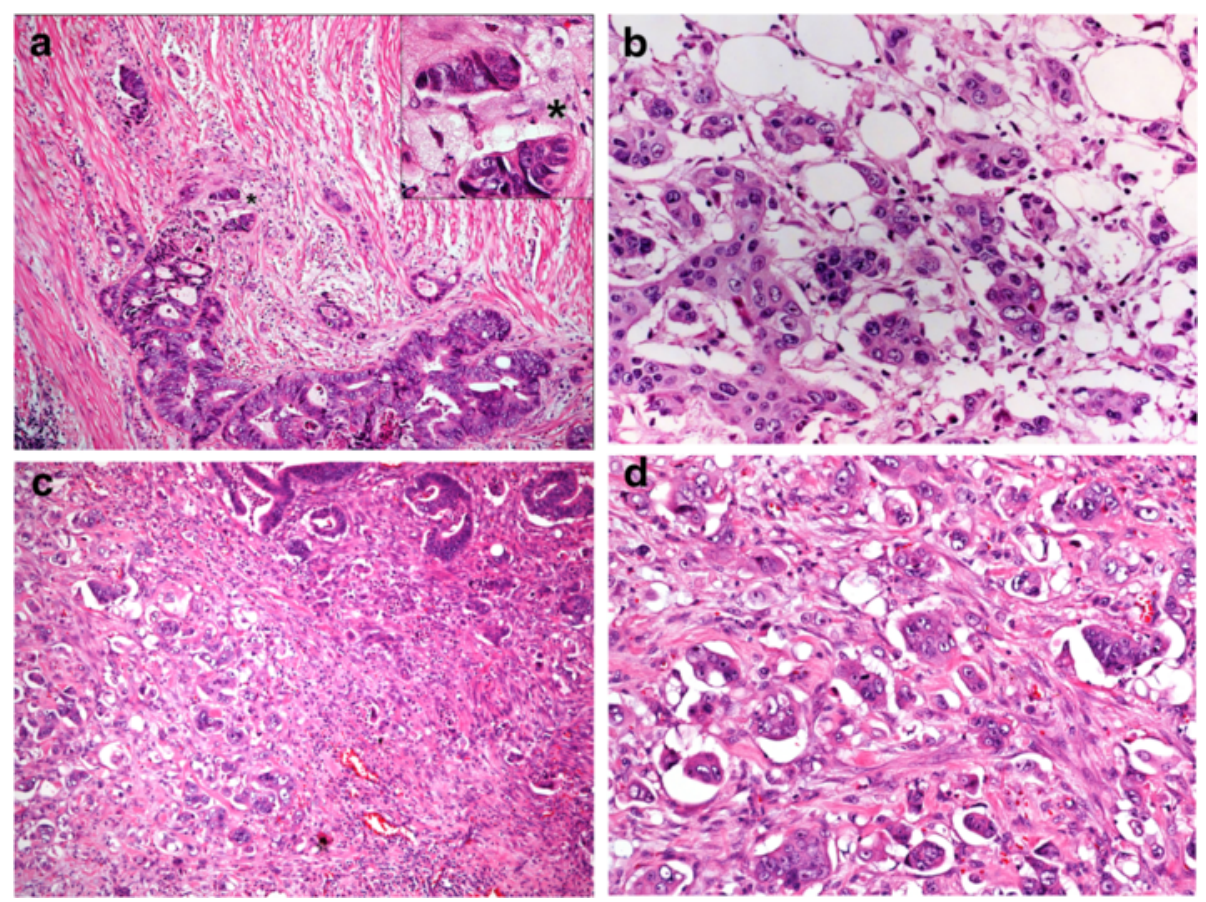

Fig. 1 Grades of malignancy of CRC based on the highest PDC count. a PDC G1: $<5$ clusters [H\&E stain 10x]. The particular of the clusters showing more than 5 undifferentiated cancer cells [asterisk - H\&E stain 20x]. b PDC G2: 5-9 clusters [H\&E stain 20x]. c PDC G3: $\geq 10$ clusters at the fron of the tumor mass [H\&E stain 10X]. $\mathbf{d}$ PDC G3: The same clusters at major resolution [H\&E stain; 20X]

at $\mathrm{H} \& \mathrm{E}$ stain and do not require the use of auxiliary immunohistochemical stains, such as cytokeratins. On the other hand, immunohistochemistry is mandatory for correct tumor budding identification, especially when it can be masqueraded by peritumoral desmoplastic tissue or inflammatory cell accumulation, as in leukocyte-rich peritumoral stroma [10-12].

The lack of information regarding PDC development has allowed for different hypothesis regarding their pathogenesis to be made. Their similar morphology suggests that PDC and tumor budding could represent sequential steps in tumor growth. This hypothesis is supported by the evidence of both PDC and tumor budding in the same tumor mass. This may serve as proof of possible sequential transformation of the latter into the former. Several studies in vitro have shown that tumor cells, singly or in large aggregates, can detach from the tumor mass and migrate into desmoplastic extracellular matrix with cohort-migration or through a mesenchymal-amoeboid transformation, activating an epithelial-mesenchymal transition process [13-19]. According to this development, PDC could represent the evolution of tumor buds or tumor podia, while they acquire proliferative and aggregative strength. Thus, PDC have been strongly associated with the up-regulation of Wnt/beta-catenin signaling pathways, such as metalloproteinase, disintegrin and L1-cell-adhesion-molecule (LICAM) $[8,20,21]$ or beta-catenin $[8,20-22]$ as well as with the loss of pro-adhesion proteins such as caderin $E[22,23]$ or claudin [24]. These findings encourage the considerazion of PDC as directly involved in tumor dissemination and metastasis formation through direct invasion of lymph vascular channels. PDC could consequently assume a fundamental role in the definition of cancer aggressiveness and prediction of tumor behavior.

Recent studies have shown that PDC are strongly associated with vascular lymph invasion and lymph nodal metastases. Furthermore, they predict N+ status with higher sensitivity and specificity compared to other traditional, unfavorable histological factors of prognosis [25-27]. This data has been demonstrated in all TNM stages, including the early (pT1) CRC where PDC are correlated with tumor depth, particularly sub-mucosal invasion $\geq 1000 \mu \mathrm{n}[27,28]$.

These results encourage considering the presence and number of PDC as possible tools in risk assessment pertaining to nodal involvement. This is especially useful when conservative, local excision of low rectal carcinoma is used, when the lymph nodes cannot be examined or the number of examined lymph nodes is less than 12 (the minimum recommended for accurate staging in CRC) [27]. Furthermore, PDC grade has been reported as a significant predictor of occult micrometastases, defined as small metastatic deposits measuring less than $2 \mathrm{~mm}$ in a greater diameter detectable by immunohistochemistry or reverse transcription polymerase chain reaction in otherwise node 
negative (pN0) tumors [9, 26-29]. In particular, it has been demonstrated that PDC grade is significantly associated with the presence of lymph node micrometastases and may therefore be used in case selection cases for timeconsuming, costly evaluation of occult nodal micrometastasis [27].

PDC's efficacy in categorizing patients affected by CRC for prognosis has been evaluated in different studies. PDC grading has been demonstrated as a strong, independent prognostic factor in CRC [12, 26-29], where the high number of PDC (PDC G2 CRC and PDC G3 CRC) is strongly predictive of short, disease free survival and short disease specific survival, independent of pTNM stages [7, 28] and other unfavorable histological features, including traditional conventional WHO grading [7, 8, 26-29].

PDC-grading appeared more accurate in identifying stage I, subgroup with poor survival. Indeed, patients with stage I PDC-rich tumors have been shown to have an overall survival comparable to, or worse than that observed in subjects with stage III CRCs $[9,26,27]$. This data is relevant in oncological practice for this category of patients as well as in stage IIA subjects, in whom the post-surgical application of chemotherapy is controversial [25, 26].

Tumor grading based on PDC counting is applicable in more than $90 \%$ of CRC endoscopic biopsies. It can reveal relevant information about the anatomical extent of the disease as well as biological proprieties of the tumor. When compared to traditional tumor grading, PDC-grading is more accurate in this context. In particular, high numbers of PDC in biopsy specimens are strongly predictive of unfavorable histological features, including infiltrating tumor borders, tumor budding, lymph vascular invasion, and perineural invasion in resection specimens, suggesting more aggressive behavior [25]. Similar to that observed in surgical specimens, a positive inter-observer agreement may be reached in the assessment of histological PDC grading in biopsy, which is higher than that achieved by using a conventional grading system [29]. These results may have remarkable clinical relevance in the choice of therapeutic management for patients affected by CRC, with particular regards to those with rectal cancer. Indeed, tumors with a low PDC grade could be submitted to local excision, whereas more invasive procedures, including anterior resection, might be reserved for those displaying a high number of PDC [30,31].

Although PDC counting in biopsy has revealed a significant correlation with the corresponding surgical specimen that would suggest a lower number, there have been criticisms that the number of detectable clusters at biopsy may appear less since samples may not be taken from the deepest part of the tumor. Other problems encountered in PDC counting in biopsies are high tissue fragmentation, possible extensive necrosis, mainly in ulcerated tumors. Beyond these criticisms, thermal electro coagulation-induced cytological artifacts may reduce the quantity of evaluable cancer tissue and induce difficulties in PDC detection $[25,29]$. The main difficulty is the distinction of PDC from transversally cut glandular crypts. These conditions increase confounding features and provoke discordance in PDC evaluation. However, bias can be avoided by excluding necrotic areas from the count and carefully considering all fragmented cluster cells detached from the sample using a critical approach.

A high PDC count has been significantly associated with CRC's mutational status; in particular with KRAS mutation. The most frequent mutations have been observed in G12A and G12C in codon 12, and G13C and G13D in codon 13. These data give insight into the mechanisms of PDC development supposing that KRAS mutations might be responsible for their formation. The association of a KRAS mutation and high PDC grade, a higher depth colonic wall invasion and development of nodal metastases may account for a poorer prognosis of KRAS mutated CRC [32]. Although with no statistical significance, a similar trend has been viewed with V600E BRAF mutation.

\section{Conclusion}

Due to its positive reproducibility and ease of histological detection, PDC are destined to assume more and more relevance in the histopathological description of $\mathrm{CRC}$, as well as in clinical and oncological practice. Although no worldwide consensus has been reached thus far, PDC may foreseeable be introduced in a histological report, considered with other, commonly noted unfavorable histological features, to give further, important prognostic information for patient management.

\section{Ethical approval and consent to participate Not applicable.}

\section{Consent to publication \\ Not applicable.}

\section{Availability of data and material \\ Not applicable.}

\section{Abbreviations}

CRC: Colorectal cancer; G1: Grade 1; G2: Grade 2; G3: Grade 3; H\&E: Haematoxylin and eosin; LICAM: L1-cell-adhesion-molecule; PDC: Poorly differentiated cluster; TNM: Tumor, node, metastasis.

\section{Competing interests}

The authors declare that they have no competing interests.

\section{Authors' contributions}

LRB conceived of the study. VB participated in the design of the study. SB participated in the design of the study and helped to draft the manuscript. FD conceived of the study and helped to draft the manuscript. CP participated in the design of the study and helped to draft the manuscript. All authors read and approved the final manuscript. 


\section{Acknowledgments}

We are grateful to the staff of the Department of Diagnostic Medicine and Public Health of Modena (Sections of Pathology and Internal medicine) as well as of the Department of Pathology of Messina.

\section{Funding}

This paper was not financially supported.

\begin{abstract}
Author details
${ }^{1}$ Department of Diagnostic Medicine and Public Health, University of Modena and Reggio Emilia - Section of Pathology, Via del Pozzo, 41124 Modena, Italy. ${ }^{2}$ Department of Pathology, University of Messina, Via Consolare Valeria, 98125 Messina, Italy. ${ }^{3}$ Department of Diagnostic Medicine and Public Health, University of Modena and Reggio Emilia - Section of Internal Medicine, Via del Pozzo, 41124 Modena, Italy. ${ }^{4}$ CURML, chemin de la Vulliette 4, 1000, Lausanne 25, Switzerland.
\end{abstract}

Received: 18 November 2015 Accepted: 10 March 2016 Published online: 22 March 2016

\section{References}

1. Kinoshita O, Kishimoto M, Murayama Y, Kuriu Y, Nakanishi M, Sakakura C, et al. The number of metastatic lymph nodes exhibiting poorly differentiated clusters predicts survival in patients with pStage III colorectal cancer. Int J Colorectal Dis. DOI 10.1007/s00384-015-2393-5.

2. Ferlay J, Shin HR, Bray F, Forman D, Mathers C, Parkin DM. Estimates of worldwide burden of cancer in 2008: GLOBOCAN 2008. Int J Cancer. 2010;127:2893-917.

3. Barresi V, Reggiani Bonetti L, leni A, Domati F, Tuccari G. Prognostic significance of grading based on the counting of poorly differentiated clusters in colorectal mucinous adenocarcinoma. Hum Pathol. 2015;46:1722-9.

4. Barresi V, Reggiani Bonetti L, leni A, Branca G, Tuccari G. Histologic prognostic markers in stage IIA colorectal cancer: a comparative study. Scand J Gastroenterol. 2016;51:314-20.

5. Hamilton SR, Bosman FT, Boffetta P, Ilyas M, Morreau H, Nakamura S-I, et al. Carcinoma of the colon and rectum. In: Bosman TF, Carneiro F, Hruban RH, Theise ND, editors. WHO Classification of Tumours of the Digestive System. Lyon: IARC Press; 2010. p. 138-9.

6. Chandler I, Houlston RS. Interobserver agreement in grading of colorectal cancers-findings from a nationwide web-based survey of histopathologists. Histopathology. 2008:52:494-9.

7. Ueno H, Kajiwara $Y$, Shimazaki H, Shinto E, Hashiguchi $Y$, Nakanishi K, et al. New criteria for histologic grading of colorectal cancer. Am J Surg Pathol. 2012;36:193-201.

8. Ueno H, Hase K, Hashiguchi $Y$, Shimazaki H, Tanaka M, Miyake O, et al. Site-specific tumor grading system in colorectal cancer: multicenter pathologic review of the value of quantifying poorly differentiated clusters. Am J Surg Pathol. 2014;38:197-204

9. Barresi V, Branca G, Vitarelli E, Tuccari G. Micropapillary pattern and poorly differentiated clusters represent the same biological phenomenon in colorectal cancer: a proposal for a change in terminology. Am J Clin Pathol. 2014;142:375-83.

10. Ueno H, Murphy J, Jass JR, Michizuki H, Talbot IC. Tumor budding as an index to estimate the potential of aggressiveness in rectal cancer. Histopathology. 2002;40:127-32.

11. Koelzer VH, Zlobec I, Berger MD, Cathomas G, Dawson H, Dirschmid K, et al. Tumor budding in colorectal cancer revisited: results of a multicenter interobserver study. Virchows Arch. 2015;466:485-93.

12. Kim JW, Shin MK, Kim BC. Clinicopathologic impacts of poorly differentiated cluster-based grading system in colorectal carcinoma. J Korean Med Sci. 2015:30:16-23.

13. Prall F, Ostwald C. High-degree tumor budding and podia-formation in sporadic colorectal carcinomas with K-ras gene mutations. Hum Pathol. 2007;38:1696-702

14. Prall F. Tumour budding in colorectal carcinoma. Histopathology. 2007;50:151-62.

15. Friedl $P$, Wolf $K$. Tumour-cell invasion and migration: diversity and escape mechanisms. Nat Rev Cancer. 2003;3:362-74.
16. Ueno H, Shinto E, Kajiwara Y, Fukazawa S, Yamamoto J, Hase K. Prognostic impact of histological categorization of epithelial-mesenchymal transition in colorectal cancer. Br J Cancer. 2014;111:2082-90.

17. Hanahan D, Weinberg RA. Hallmarks of cancer: the next generation. Cell. 2011;144:646-74.

18. Brabletz T, Jung A, Spaderna S, Hlubek F, Kirchner T. Migrating cancer stem cells-an integrated concept of malignant tumor progression. Nat Rev Cancer. 2005;5:744-9.

19. Karagiannis GS, Poutahidis T, Erdman SE, Kirsch R, Riddell RH, Diamandis EP. Cancer-associated fibroblasts drive the progression of metastasis through both paracrine and mechanical pressure on cancer tissue. Mol Cancer Res. 2012;10:1403-18.

20. Kevans D, Wang LM, Sheahan K, Hyland J, O'Donoghue D, Mulcahy H, et al. Epithelial-mesenchymal transition (EMT) protein expression in a cohort of stage II colorectal cancer patients with characterized tumor budding and mismatch repair protein status. Int J Surg Pathol. 2011;19:751-60.

21. Kajiwara $Y$, Ueno $H$, Hashiguchi $Y$, Shinto $E$, Shimazaki $H$, Mochizuki $H$, et al. Expression of I1 cell adhesion molecule and morphologic features at the invasive front of colorectal cancer. Am J Clin Pathol. 2011;136:138-44.

22. Kajiwara $Y$, Ueno $H$, Hashiguchi $Y$, Shinto E, Shimazaki H, Mochizuki H, et al. Heterogeneity of metalloproteinase expression in colorectal cancer - relation of molecular findings to basic morphology. Anticancer Res. 2011:31:1567-75.

23. Kalluri R, Weinberg RA. The basics of epithelial-mesenchymal transition. J Clin Invest. 2009:119:1420-8.

24. Shibutani M, Noda E, Maeda K, Nagahara H, Ohtani H, Hirakawa K. Low expression of claudin-1 and presence of poorly-differentiated tumor clusters correlate with poor prognosis in colorectal cancer. Anticancer Res. 2013;33:3301-6.

25. Barresi V, Bonetti LR, leni A, Branca G, Baron L, Tuccari G. Histologic grading based on counting poorly differentiated clusters in preoperative biopsy predicts nodal involvement and pTNM stage in colorectal cancer patients. Hum Pathol. 2014;45:268-75.

26. Barresi V, Branca G, leni A, Reggiani Bonetti L, Baron L, Mondello S, et al Poorly differentiated clusters (PDCs) as a novel histological predictor of nodal metastases in pT1 colorectal cancer. Virchows Arch. 2014:464:655-62.

27. Barresi V, Reggiani Bonetti L, Branca G, Di Gregorio C, Ponz de Leon M, Tuccari G. Colorectal carcinoma grading by quantifying poorly differentiated cell clusters is more reproducible and provides more robust prognostic information than conventional grading. Virchows Arch. 2012;461:621-8.

28. Ueno H, Hase K, Hashiguchi Y, Shimazaki H, Yoshii S, Kuod SE, et al. Novel risk factors for lymph node metastasis in early invasive colorectal cancer: a multi-institution pathology review. J Gastroenterol. 2014;49:1314-23.

29. Barresi V, Tuccari G. Colorectal carcinoma grading quantified by counting poorly differentiated clusters: is it feasible on endoscopic biopsies? Am J Surg Pathol. 2013;37:943-5

30. Nakadoi K, Tanaka S, Kanao H, Terasaki M, Takata S, Oka S, et al. Management of T1 colorectal carcinoma with special reference to criteria for curative endoscopic resection. J Gastroenterol Hepatol. 2012;27:1057-62.

31. Tytherleigh MG, Warren BF, Mortensen NJ. Management of early rectal cancer. Br J Surg. 2008;95:409-23.

32. Barresi $V$, Reggiani Bonetti L, Bettelli S. KRAS, NRAS, BRAF mutations and high counts of poorly differentiated clusters of neoplastic cells in colorectal cancer: observational analysis of 175 cases. Pathology. 2015;47:551-6.

\section{Submit your next manuscript to BioMed Central and we will help you at every step:}

- We accept pre-submission inquiries

- Our selector tool helps you to find the most relevant journal

- We provide round the clock customer support

- Convenient online submission

- Thorough peer review

- Inclusion in PubMed and all major indexing services

- Maximum visibility for your research

Submit your manuscript at www.biomedcentral.com/submit 